\title{
Clinical characteristics of HIV-negativepatients with cryptococcemia in a Chinese tertiary-care hospital: $\llbracket$ A 10-year study
}

\section{Liling Liang}

Chinese PLA General Hospital

\section{Zhixin Liang}

Chinese PLA General Hospital

Liang-an Chen ( $\nabla$ chenliangan301@163.com )

the Chinese People's Liberation Army General Hospital https://orcid.org/0000-0002-7373-0595

\section{Research}

Keywords: cryptococcemia, Cryptococcus neoformans, clinical characteristics, diagnosis, treatment

Posted Date: March 27th, 2020

DOI: https://doi.org/10.21203/rs.3.rs-17744/v1

License: (c) (i) This work is licensed under a Creative Commons Attribution 4.0 International License.

Read Full License 


\section{Abstract}

Objective: Cryptococcosis is a severe opportunistic fungal infection. Cryptococcemia is a rare manifestation of cryptococcosis with high mortality. Objective: To characterize patients with cryptococcemia in Chinese PLA General Hospital since 2010 to 2019.

Methods: We identified retrospectively those blood cultures with Cryptococcus sp. growth, and then obtained their clinical information which were analyzed by two independent reviewers.

Results: A total of 8 patients withcryptococcemiawere identified, of which only 2 patients survived. There were 3 males and 5 females, whose age ranged from 23 to 72 years. They were all HIV negative. 1 (12.5\%) had ovarian adenocarcinoma, 2 (25\%) had systemic lupus erythematosus, 3 (37.5\%) had primary nephrotic syndrome, 1 (12.5\%) had viral myocarditis, and $1(12.5 \%)$ were after lung transplantation. The most common symptoms were fever (100\%) and confusion (50\%). Out of the 8 episodes, $6(60 \%)$ had meningeal infection and $2(25 \%)$ presented as pulmonary involvement meanwhile. Six patients were dead at half a year follow up.

Conclusions: Cryptococcemia is an unusual infection characterized by a high mortality. It requires early identify and prompt antifungal therapy.

\section{Background}

Cryptococcosis is an opportunistic fungal infection caused by the encapsulated yeasts of genus Cryptococcus. This fungus particularly affects immunocompromised individuals especially among patients with human immunodeficiency virus (HIV) infection and AIDS. Meningoencephalitis and pulmonary infiltrates are the two most common presentations of cryptococcosis. Cryptoccemia is uncommon but severe form of invasive cryptococcal diseases. There were still few studies to describe the full characteristics of this fulminant condition specifically ${ }^{[1-3]}$ except some clinical case series. Hence we conducted this retrospective study to analyze the clinical features and therapeutic outcomes of patients with cryptococcemia in a large tertiary-care hospital in China, hoping to delineate the clinical characteristics of this unusual infection.

\section{Methods}

\section{Hospital setting}

This study was performed at the First Medical Centre of Chinese People's Liberation Army General Hospital (PLAGH) (Peking, China), a large tertiary-care hospital with a 3000-bed capacity and 190 thousand inpatients per year in northern China. The hospital provides medical service for both the military and the public.

\section{Study design}


We searched the database of the Infection Management and Disease Control Department of the First Medical Centre of PLAGH for Cryptococcus spp. isolated from blood since January $1^{\text {st }} 2010$ to December $31^{\text {st }} 2019$. The medical records of these patients were reviewed and analyzed. The duration from admission to diagnosis was calculated. The clinical data collected included demographics, underlying conditions, interval from blood drawing to reporting, initial clinical symptoms, risk factors, laboratory examinations, sites of involvement, Sequential Organ Failure Assessment (SOFA) score, treatments and prognosis. All records were analyzed by two independent reviewers.

\section{Definitions}

Cryptococcemia was defined as one or more positive blood cultures growing Cryptococcus spp. in this study. The time of diagnosis was defined as the day when positive result was reported. The SOFA score was calculated according to the results of several assay tests at the day of blood collected. Antimicrobial therapy was considered empirical when the antibiotic was used before the results of antimicrobial susceptibility test return. Pulmonary involvement was considered if the patient had compatible abnormal computed tomography (CT) scan or chest X-ray presentation. The effective anticryptococcal treatment was defined as the patient received fluconazole, itraconazole, voriconazole, or amphotericin B for more than 72 hours.

\section{Statistical analysis}

Descriptive statistic data were presented as means, range and standard deviation for continuous variables and as frequencies and percentages for categorical variables. Analyses were performed using the SPSS software, version 20.0.

\section{Results}

\section{Characteristics of the patients}

During the 10-year period, we screened 10,211 blood stream infections and a total of 8 patients with cryptococcemia were identified. Characteristics of the patients with cryptococcemia were shown in Table 1. Of them, the ages ranged from 23 to 72 years. 3 (37.5\%) patients were male. The body mass index (BMI) was within the normal range. The mean SOFA score was $3.25 \pm 3.8$. They were all HIV negative. The most common underlying disease was primary nephrotic syndrome (37.5\%), followed by systemic lupus erythematosus (SLE) (25\%). 1 (12.5\%) had ovarian adenocarcinoma, 1 (12.5\%) were after lung transplantation and 1 (12.5\%) had viral myocarditis without obvious defect of immunity. Fever (100\%) was the most common symptom of these patients, followed by confusion $(50 \%)$ and headache $(25 \%)$. There was 1 (12.5\%) patient suffered from nausea/vomiting, seizure and cough/expectoration, respectively. All patients received multiply antibiotics therapy and $6(75 \%)$ accepted glucocorticoids or immunosuppressants treatment before identification of cryptococcal infection. 4 (50\%) patients received central vein catheterization. None had history of pigeons contact. The mean white blood cell (WBC) count of the patients was $10.54 \pm 3.27 \times 10^{9} / \mathrm{L}$, of which the percentage of neutrophils (NEUT\%) was $76.35 \pm$ 
0.27. The mean C-reaction protein (CRP) concentration was $4.15 \pm 4.27 \mathrm{mg} / \mathrm{dl}$, which was beyond the normal range $(0-0.8 \mathrm{mg} / \mathrm{dl})$. Polymicrobial bloodstream infection was occurred in $2(25 \%)$ patients. All patients received chest CT scan or X-ray examination and 2(25\%) patients presented consolidation and reticular opacity on CT imaging. There were 2 patients received brain magnetic resonance imaging (MRI) scan and brain CT scan respectively but showed no significant sign of meningeal infection. Another 4 patients didn't receive any brain imaging examination. 1 (12.5\%) patient received lumbar puncture examination and $C$. neoformans was identified in cerebrospinal fluid culture. Although most patients didn't receive lumbar puncture examination, 6 patients showed symptoms of central nerve system (CNS) such as headache, confusion excluded other reasons. These $6(75 \%)$ patients were considered as meningeal involvement. $2(25 \%)$ patients received serum cryptococcal antigen test and both showed a positive result. The interval of diagnosis was $15.4 \pm 6.02$ days. The interval of blood culture report was $7.5 \pm 1.73$ days.

\section{Antifungal susceptibility test}

2 of the 8 patients showed two positive blood culture results. 1 patient showed positive results of both blood culture and cerebrospinal fluid (CSF) culture. The data was counted only once in the results of demographic and clinical characteristics but was included in the results of antifungal drugs susceptibilities. Thus, 11 clinical isolates were included. 11 isolates were all identified as $\mathrm{C}$. neoformans var. grubii by IGS sequence analysis. The results of antifungal susceptibility test of 1 isolate was found to be lost. As a result of that, only 10 isolates were available for antimicrobial susceptibility analysis. The results were presented in Table 2. 10 clinical strains of Cryptococcus were sensitive to fluconazole, voriconazole, itraconazole, amphotericin B and flucytosine in vitro.

\section{Treatment and prognosis}

As Table 3 presented, 1 patient (patient 1) died before establishment of the diagnosis of cryptococcemia and did not receive any antifungal treatment. 1 patient (patient 6) was diagnosed one day before her dead and only received one dose voriconazole. $6(75 \%)$ patients received effective anticryptococcal treatment. Of these, 3 patients received antifungal treatment with voriconazole for 10,14 and 47 days respectively until their dead. 1 patient received combination antifungal treatment with voriconazole and flucytosine. He died after 2 months because of multiple organ failure. 1 patient received fluconazole sequential voriconazole treatment for 1 and a half years. He has been followed for 3 years without relapse. Another patient received combination antifungal treatment with fluconazole and amphotericin B. $\mathrm{He}$ is continuing oral fluconazole for maintenance treatment at present. Of the 2 surviving patients, none developed neurological sequelae.

\section{Discussion}

In this study, we retrospectively analyzed the clinical features of 8 patients with cryptococcemia. This is a rare blood stream infection. It is a first study of cryptococcemia reported in north China as we know by now. It is consistent with the phenomenon that the incidence of cryptococcosis in north China is less than 
which in south China ${ }^{[4-6]}$. The study showed the characterization of patients in whom cryptococcemia was the initial diagnostic proof of cryptococcosis. Meanwhile, it also provided information about the microbial features of Cryptococcus spp. isolated from blood.

As a sign of graveness, cryptococcemia was mostly developed in immunocompromised hosts especially in patients with late-stage HIV infection ${ }^{[1]}$. In addition to HIV infection, solid-organ transplantation, immunosuppressive medications treatment, chronic renal failure, hematologic malignancy, severe hepatic diseases and rheumatologic disorders can also predispose individuals to this infection ${ }^{[1-3]}$. In our study, $87.5 \%$ of patients had predisposing conditions. 6 patients (75\%) had been treated with corticosteroids or immunosuppressants for various reasons before identification of cryptococcal infection. It was noteworthy that 1 cryptococcemia patient with viral myocarditis was apparently immunocompetent in this study. The potential of Cryptococcus spp. to cause endovascular infection and endocarditis is very low. Interestingly, cryptococcosis occurs more frequently in immunocompetent patients than in immunocompromised patients in China ${ }^{[7-10]}$. The mechanism of this is not clear. Maybe there are immune function abnormalities in this kind of patients that cannot be recognized by laboratory tests at present. It is worth mentioning that this patient suffered multiple deep vein catheterization, which does not exclude the probability of iatrogenic bloodstream infection. In addition, it is worth noting that Cryptococcus is known to be common in excreta from certain birds such as pigeons. All patients in our study had no history of pigeon contact. Many clinicians only consider the diagnosis of cryptococcosis based the contact history with pigeons, which greatly delayed the diagnosis of cryptococcosis.

The symptoms of cryptococcemia were usually nonspecific and atypical. They were sometimes mistaken for manifestations of primary disease. These posed considerable diagnostic challenges for clinicians. Fever were found in all eight patients with cryptococcemia. Due to the unique propensity of CNS invasion of Cryptococcus, approximately $80 \%$ of cryptococcemia patients had complicated meningitis ${ }^{[1,11]}$. Clinical manifestations of CNS involvement include headache, nausea/vomiting, altered consciousness and signs of meningeal irritation. In this study most $(75 \%, 6 / 8)$ patients lacked typical symptoms of headache and nausea/vomiting. $4(50 \%, 4 / 8)$ patients presented subtle confusion. The mild symptoms of headache and nausea/vomiting may be ignored in medical daily inquiring or not described in the medical record. Therefore, the incidence of symptoms of meningitis was likely to be underestimated.

The imaging manifestations in early stage of cryptococcal meningitis were not obvious. MRI/CT scan of the brain were performed in 4 patients in our study. None showed evidence of cryptococcal infection. So negative brain imaging cannot preclude the development of disseminated cryptococcal infection. Rather than imaging examination, the test of cryptococcal polysaccharide capsular antigen (CrAg) in serum or CSF is highly sensitive and specific for disseminated Cryptococcus infection ${ }^{[12]}$. It is an early diagnosis method of cryptococcosis. And it took less time than blood culture of Cryptococcus (mean time, 7.5 days in this study). With the introduction of the point-of-care lateral flow cryptococcal antigen assay, it has been greatly facilitated with lower cost and more sensitivity than earlier serological tests ${ }^{[13,14]}$. However, it was regret that serum cryptococcal antigen assay were not performed in most patients. So the diagnosis 
of opportunistic infections including cryptococcosis should be early considered in immunocompromised patients. It may be necessary to perform serum cryptococcal antigen test in routine diagnostic work-up in high risk individuals especially with fever of unknown origin.

C. neoformans and C. gattii are the two main pathogenic cryptococcal species for humans ${ }^{[14]} .11$ Cryptococcus spp. isolates are all C. neoformans var. grubii. The data were consistent with a multicenter investigation that $\mathrm{C}$. neoformans var. grubii accounted for $97.1 \%$ of all $\mathrm{C}$. neoformans species complex in China $^{[15]} .11$ clinical strains of Cryptococcus were sensitive to fluconazole, voriconazole, itraconazole, amphotericin B and flucytosine in vitro. The results provided some information about the microbial features of Cryptococcus spp. isolated from blood. However, it is difficult to draw conclusions about the overall microbiology and antifungal drug sensitivity because of its small sample size.

Cryptococcemia is an extremely severe form of invasive cryptococcosis with poor prognosis. It had shown a high mortality rate between $30 \%$ and $40 \%$ in previous studies ${ }^{[1,2,16]}$. Effective antifungal therapy is the cornerstone to treat disseminated cryptococcosis including cryptococcemia. It was recommended that induction therapy for disseminated cryptococcosis using fungicidal regimens, such as a polyene and flucytosine, followed by consolidation and maintenance therapy using suppressive regimens with fluconazole ${ }^{[17]}$. The other triazoles including itraconazole, voriconazole and posaconazole are active against cryptococcal isolates in vitro but due to the differences in bioavailability, CSF permeability, drug interactions, cost-benefit ratio, and lack of strong studies on cryptococcosis, these agents were not recommended as first-line agents for consolidation or maintenance therapy. They were used clinically in salvage situations. While echinocandins have no in vivo activity versus Cryptococcus species. In our study, 6 patients received antifungal treatment. But only 1 patient received amphotericin B-containing regimen and flucytosine-containing regimen, respectively. Most patients didn't receive appropriate antifungal therapy. Most clinicians agreed that voriconazole was an effective treatment for cryptococcosis while neglected the fact that it was not enough in the treatment of disseminated cryptococcosis. In addition, considerable clinicians preferred conservative regimens rather than concerned about the side effects of amphotericin B and flucytosine. Both of above causes led to the inadequacy of early induction treatment of the disease.

Although cryptococcemia may have contributed to the fatal outcome of itself, we believe that the delay in the diagnosis and improper treatment is the main contributing factor. The patients first diagnosed by blood cultures had mild and neglected symptoms of meningitis, which may lead to the delayed diagnosis. These patients also had less access to effective antifungal treatment. It caused poor outcome. This report highlighted the need to early identify this rare infectious disease and its clinical presentations, especially in immunocompromised patients. The results further highlighted the important of prompt aggressive interventions in improving outcome of cryptococcemia. However, the research has several limitations. Although we collected 10-year data of cryptococcemia in PLAGH, the sample size of this research is still small due to the low incidence of cryptococcemia. Another limitation is the absent usage of latex agglutination and lateral-flow assay for CrAg determination. Furthermore, the antifungal susceptibility of Cryptococcus may vary among different hospitals, especially those in different regions. 
Therefore, prospective studies involving larger sample size and more hospitals are needed to provide more information about cryptococcemia.

\section{Conclusion}

This study identified the prevalence of $\mathrm{C}$. neoformans var. grubii causing cryptococcemia in Peking, Northern China. This rare infection usually happens in immunocompromised patients and is characterized by a high mortality rate. The diagnosis of cryptococcemia should be early considered in these patients especially with fever of unknown origin. It is apparent that insightful effective anticryptococcal therapy is crucial to patient's prognosis for those with cryptococcemia.

\section{Abbreviations}

HIV: human immunodeficiency virus; PLAGH: Chinese People's Liberation Army General Hospital; SOFA: Sequential Organ Failure Assessment; CT: computed tomography; BMI: body mass index; SLE: systemic lupus erythematosus; WBC: white blood cell; CRP: C-reaction protein; MRI: magnetic resonance imaging; CNS: central nerve system;CSF: cerebrospinal fluid; CrAg: cryptococcal polysaccharide capsular antigen

\section{Declarations}

\section{Acknowledgements}

We thank all the colleagues working on the ongoing prospective health care-associated infection surveillance electronic database in the PLAGH hospital. Without their prior efforts, this study could not be possible.

\section{Funding}

This work was supported by grants from Military Medical Science and Technology Innovation Subject of China (16CXZ041).

\section{Availability of data and materials}

The data generated or analysed during this study are not publicly available to avoid disclosure the individual of the patients, but all data are available from the corresponding author on reasonable request.

\section{Authors' contributions}

LC conducted the design of this study, revised the manuscript and was responsible for the manuscript. LL and $\mathrm{ZL}$ collected the clinical data, interpreted the results and wrote the manuscript. All authors read and approved the final manuscript.

\section{Ethics approval and consent to participate}


This study was approved by the Clinical Trial Ethics Review Committee of the PLAGH Hospital. For this type of retrospective study formal consent is not required.

Consent for publication

Not applicable.

Competing interests

The authors declare that they have no competing interests.

Publisher's Note

Springer Nature remains neutral with regard to jurisdictional claims in published maps and institutional affiliations.

\section{Author details}

Department of Respiratory Medicine, the First Medical Centre of Chinese PLA General Hospital, Fuxing Road No. 28, Beijing 100853, China.

\section{References}

1. Brizendine KD BJaPP. Predictors of mortality and differences in clinical features among patients with Cryptococcosis according to immune status. PLOS ONE 2013; 8(3):e60431.

2. Chen J VA, Diaz MR, Litvintseva AP, Wollenberg KK, Kwon-Chung KJ. Cryptococcus neoformans strains and infection in apparently immunocompetent patients, China. Emerg Infect Dis 2008; 14(5):755-762.

3. Fan XXM, Chen S, et al. . Predominance of Cryptococcus neoformans var. grubii multilocus sequence type 5 and emergence of isolates with non-wild-type minimum inhibitory concentrations to fluconazole: a multi-centre study in China. Clin Microbiol Infect 2016; 22(10):e881-887.

4. Fu Y XM, Zhou H, Yao Y, Zhou J, Pan Z. . Microbiological and clinical characteristics of cryptococcemia: a retrospective analysis of $\mathbf{8 5}$ cases in a Chinese hospital. Medical Mycology 2019:1-7.

5. J. N. Jarvis AP, S. Bauman et al. Evaluation of a novel point-of-care cryptococcal antigen test on serum, plasma, and urine from patients with HIV-associated cryptococcal meningitis. Clin Infect Dis 2011; 53(10):1019-1023.

6. Jean SS FC, Shau WY, Chen YC, Chang SC, Hsueh PR, Hung CC, Luh KT. Cryptococcaemia: clinical features and prognostic factors. QJM 2002; 95(8):511-518.

7. Kohno S KH, Izumikawa $\mathrm{K}$, et al. . Clinical features of pulmonary cryptococcosis in non-HIV patients in Japan. $J$ Infect Chemother 2015; 21(1):23-30. 
8. Li A P, W., Wu, S., et al. Ecological surveys of the Cryptococcus species complex in China. Chin Med J 2012; 125(3):511-516.

9. Lin L T, Y., Zhu, et al. Initial research of phenotypic and molecular characteristics of 80 Cryptococcus neoformans environmental strains from China mainland. J Pract Dermato/ 2013; 6(3):71-73.

10. Liu K DH, Xu B, You R, Xing Z, Chen J, Lin Q, Qu J. Clinical analysis of non-AIDS patients pathologically diagnosed with pulmonary cryptococcosis. J Thorac Dis 2016; 8(10):2813-2821.

11. Pasqualotto AC BSC, de Mattos Oliveira F, Severo LC. Cryptococcemia: an analysis of $\mathbf{2 8}$ cases with emphasis on the clinical outcome and its etiologic agent. Rev Iberoam Micol 2004; 21(3):143-146.

12. Perfect JR DD, Gallis HA. Cryptococcemia. Medicine 1983; 62(2):98-109.

13. Perfect JR DW, Dromer F, Goldman DL, Graybill JR, Hamill RJ, Harrison TS, Larsen RA, Lortholary O, Nguyen MH, Pappas PG, Powderly WG, Singh N, Sobel JD, Sorrell TC. Clinical practice guidelines for the management of cryptococcal disease: $\mathbf{2 0 1 0}$ update by the Infectious Diseases Society of America. Clin Infect Dis 2010; 50(3):291-322.

14. Riha RL PA. BMJ best practice: cryptococcosis. Br Med Journals 2018.

15. Srikanta D S-TF, Doering TL. Cryptococcus neoformans: Historical curiosity to modern pathogen. Yeast 2014; 31(2):47-60.

16. Wu S G, N., Li, X., et al. . Human pathogenic fungi in China - emerging trends from ongoing national survey for 1986, 1996, and 2006. Mycopathologia 2011; 171(6):387-393.

17. Xie X XB, Yu C, et al. Clinical analysis of pulmonary cryptococcosis in non-HIV patients in south China. Int J Clin Exp Med 2015; 8:3114-3119.

\section{Tables}

Table 1 Demographic and clinical characteristics of the patients with cryptococcemia 


\begin{tabular}{|c|c|}
\hline Characteristics & Patients with cryptococcemia $(\mathrm{N}=8)(\mathrm{n}, \%)$ \\
\hline \multicolumn{2}{|l|}{ Patients demographics } \\
\hline Age (yr), mean \pm SD (range) & $43.1 \pm 13.1(23-72)$ \\
\hline Male sex & $3(37.5)$ \\
\hline BMI, mean \pm SD (range) & $22.36 \pm 3.47(17.1-28)$ \\
\hline \multicolumn{2}{|l|}{ Underlying condition } \\
\hline Malignancy & $1(12.5)$ \\
\hline Lung transplantation & $1(12.5)$ \\
\hline Systemic lupus erythematosus & $2(25)$ \\
\hline Primary nephrotic syndrome & $3(37.5)$ \\
\hline Viral myocarditis & $1(12.5)$ \\
\hline \multicolumn{2}{|l|}{ Risk factors } \\
\hline History of pigeon contact & 0 \\
\hline Central venous catheterization & $4(50)$ \\
\hline GCS or immunosuppressants treatment & $6(75)$ \\
\hline Multiply antibiotics therapy & $8(100)$ \\
\hline \multicolumn{2}{|l|}{ Symptoms } \\
\hline Fever & $8(100)$ \\
\hline Headache & $2(25)$ \\
\hline Nausea/Vomiting & $1(12.5)$ \\
\hline Confusion & $4(50)$ \\
\hline Seizure & $1(12.5)$ \\
\hline Cough/Expectoration & $1(12.5)$ \\
\hline \multicolumn{2}{|l|}{ Sites of involvement } \\
\hline Central nervous system & $6(75)$ \\
\hline Lung & $2(25)$ \\
\hline \multicolumn{2}{|l|}{ Diagnostic related information } \\
\hline Days from admission to diagnosis of cryptococcemia, mean \pm SD (range) & $15.4 \pm 6.02(7-25)$ \\
\hline Days of blood culture report, mean \pm SD (range) & $7.5 \pm 1.73(4-10)$ \\
\hline Polymicrobial bloodstream infection & $2(25)$ \\
\hline SOFA score, mean \pm SD (range) & $3.25 \pm 3.8(0-11)$ \\
\hline \multicolumn{2}{|l|}{ Treatment } \\
\hline Effective anticryptococcal treatment & $6(75)$ \\
\hline Amphotericin B-containing regimen & $1(12.5)$ \\
\hline \multicolumn{2}{|l|}{ Outcome } \\
\hline survive & $2(12.5)$ \\
\hline
\end{tabular}

Table 2 The susceptibilities of the 11 Cryptococcus spp. isolates to antifungal drugs (ATB FUNGUS 3) 


\begin{tabular}{|c|c|c|c|c|c|c|c|}
\hline \multirow[t]{2}{*}{ Patients } & \multirow[t]{2}{*}{ Gender } & \multirow[t]{2}{*}{ Age } & \multicolumn{5}{|c|}{$\mathrm{MIC}$ range $(\mathrm{mg} / \mathrm{L})$} \\
\hline & & & FCZ & $\mathrm{ICZ}$ & VRC & $\mathrm{AMB}$ & $5-\mathrm{FC}$ \\
\hline \multirow[t]{2}{*}{1} & $\mathrm{~F}$ & 41 & $<1$ & $<0.125$ & $<0.06$ & $<0.5$ & $<4$ \\
\hline & & & $<1$ & $<0.125$ & $<0.06$ & $<0.5$ & $<4$ \\
\hline 2 & $\mathrm{~F}$ & 41 & 2 & $<0.125$ & $<0.06$ & $<0.5$ & $<4$ \\
\hline \multirow[t]{2}{*}{3} & $\mathrm{~F}$ & 23 & 2 & 0.5 & 0.25 & $<0.5$ & $<4$ \\
\hline & & & 2 & 0.5 & 0.25 & $<0.5$ & $<4$ \\
\hline 4 & M & 46 & $\mathrm{~N} / \mathrm{A}$ & $\mathrm{N} / \mathrm{A}$ & $\mathrm{N} / \mathrm{A}$ & $\mathrm{N} / \mathrm{A}$ & $\mathrm{N} / \mathrm{A}$ \\
\hline \multirow[t]{2}{*}{5} & $\mathrm{~F}$ & 33 & 2 & 0.25 & 0.125 & $<0.5$ & $<4$ \\
\hline & & & 2 & 0.25 & 0.125 & $<0.5$ & $<4$ \\
\hline 6 & $\mathrm{~F}$ & 72 & 2 & $<0.125$ & $<0.06$ & $<0.5$ & $<4$ \\
\hline 7 & M & 43 & $<1$ & 0.25 & 0.125 & $<0.5$ & $<4$ \\
\hline 8 & M & 46 & $<1$ & $<0.125$ & $<0.06$ & $<0.5$ & $<4$ \\
\hline
\end{tabular}

FCZ, fluconazole; ICZ, itraconazole, VRC, voriconazole , AMB, amphotericin B, 5-FC, 5- flucytosine

N/A = Not available. The antifungal susceptibility of patient 4 could not be obtained.

Table 3 The antifungal treatment and prognosis of the patients with cryptococcemia

\begin{tabular}{lcccccc}
\hline Patients & Gender & Age & Underlying disease & Treatment before blood culture report & Treatment after blood culture report & Prognosis \\
\hline 1 & F & 41 & SLE & No & No \\
2 & F & 41 & VMC & VRC & FCZ \\
3 & F & 23 & PNS & No & VRC & VRC \\
4 & M & 46 & PNS & No & Die \\
5 & F & 33 & SLE & No & DRC+5-FC \\
6 & F & 72 & EOC & No & VRC (1 dose $)$ \\
7 & M & 43 & PNS & CAS/VRC & DRC/FCZ \\
8 & M & 36 & LTX & VRC/CAS & FCZ+AMB \\
\hline
\end{tabular}

FCZ fluconazole, ICZ itraconazole, VRC voriconazole , AMB amphotericin B, 5-FC 5- flucytosine, CAS caspofungin

VMC viral myocarditis, PNS primary nephrotic syndrome, EOC endometrioid ovarian cancer, LTX lung transplantation 